Карпенко Николай Иванович (Москва). Доктор технических наук, профессор, академик РААСН. Руководитель лаборатории НИИСФ РААСН (Научно-исследовательский институт строительной физики Российской академии архитектуры и строительных наук). Эл. почта: niisf_lab9@mail.ru.

Карпенко Сергей Николаевич (Москва). Доктор технических наук. Главный научный сотрудник НИИСФ РААСН (Научноисследовательский институт строительной физики Российской академии архитектуры и строительных наук). Эл. почта: niisf_lab9@mail.ru.

Karpenko Nikolai I. (Mosccow). Doctor of Technical Sciences, Professor. Chief Research Officer of thelaboratory at the Research Institute of Building Physics of RAACS (21 Lokomotivny proezd, Moscow, 127238. NIISF RAACS). E-mail: niisf_lab9@mail.ru.

Karpenko Sergey N. (Moscow). Doctor of Technical Sciences. Chief Researcher at the Research Institute of Building Physics of RAACS (21 Lokomotivny proezd, Moscow, 127238. NIISF RAACS). E-mail: niisf_lab9@mail.ru.

(- Карпенко Н.И., Карпенко С.Н., 2021. Academia. Архитектура и строительство, № 4, стр. 94-98.

\title{
0 двухфакторной гравитонной модели сил тяжести при малых и больших скоростях движения тел
}

Н.И.Карпенко, НИИСФ РААСН, РААСН, Москва

С.Н.Карпенко, НИИСФ РААСН, Москва

Силы тяжести (силы гравитационного, гравитонного притяжения) тел играют важную роль в различных областях науки и техники, в том числе и в строительной отрасли. Эти силы определяться на основании закона притяжения И. Ньютона. Однако физическая природа переносчика сил притяжения в этом законе остается открытой.

В работах авторов физическая природа формирования и передачи сил тяжести раскрывается на основании установленных закономерностей вхождения в массу и реактивного выброса из неё с большой скоростью потоков гравитонов (условно - за счёт гравитонного дыхания масс) с последующим рассеиванием и действием рассеянных потоков на встречные массы тел (условно на встречные тела). Показано, что взаимное действие потоков гравитонов двух тел сводится к закону тяготения И. Ньютона.

В данной статье показано развитие гравитонной модели с учётом влияния на массу двух факторов, связанных с выбрасываемыми из неё гравитонами. С одной стороны, они разуплотняют массу при движении внутри неё, а с другой уплотняют её всесторонним сжатием при выбросах.

Указанный двойственный характер меняет представление о гравитационном ускорении.

Рассмотрено развитие гравитонной модели притяжения тел на тела, движущиеся с большими скоростями. При этом закон И. Ньютона выполняется только в случае, когда скорости движения тел являются одинаковыми. Притяжение тел, движущихся с большой скоростью, другим телом равносильно увеличению массы движущихся тел по формуле Лоренца. С другой стороны, сила притяжения движущихся тел, наоборот, снижается обратно пропорционально формуле Лоренца.

При этом, согласно гравитонной модели, массы тел не изменяются, а указанное их увеличение и уменьшение связано с особенностями действия гравитонов.

Ключевые слова: силы тяжести, масса тела, потоки гравитонов, скорость потоков, реактивный выброс, силы сжатия, разуплотнение и уплотнение массы, двойственный характер сил тяжести, скорость движения тел, усиление выброса гравитонов, усиление и ослабление сил притяжения.

On the Two-Factor Graviton Model of Gravity Forces at Low and High Velocities of Motion of Bodies

N.I.Karpenko, NIISF RAACS, RAACS, Moscow

S.N.Karpenko, NIISF RAACS, Moscow

The gravity forces (the forces of gravitational attraction, gravitonic attraction) of bodies play an important role in various fields of science and technology, including the construction industry. These forces are determined on the basis of Newton'slaw of gravity. However, the physical nature of the carrier of the forces of attraction in thislaw remains open.

In the works of the authors, the physical nature of the formation and transmission of gravity forces is revealed based on the established regularities of entry into the mass and reactive ejection from it at a high speed of gravitonic flows (conditionally due to gravitonic respiration of masses), followed by scattering and the action of scattered flows on oncoming masses of bodies (conditionally on oncoming 
bodies). It is shown that the mutual action of gravitonic flows of two bodies is reduced to thelaw of gravity of I. Newton.

In this article, the development of the graviton model is given, taking into account the influence of two factors on the mass associated with the gravitons ejected from it. On the one hand, they decompress the mass when moving inside it, and on the other, they compact it with a comprehensive compression during ejections.

This dual character changes the idea of gravitational acceleration.

The development of the graviton model of attraction of bodies to bodies moving at high speeds is considered. At the same time, Newton'slaw is fulfilled only in the case when the speeds of the bodies are the same. The attraction of bodies moving at high speed by another body is equivalent to an increase in the mass of moving bodies according to the Lorentz formula. On the other hand, the force of attraction of moving bodies, on the contrary, decreases inversely proportional to the Lorentz formula.

At the same time, according to the graviton model, body masses do not change, and their increase and decrease are associated with the peculiarities of the action of gravitons.

Keywords: gravity, body mass, flows of gravitons, speed of flows, jet ejection, compressive forces, decompression and compaction of mass, the dual nature of gravity, speed of movement of bodies, increase in the release of gravitons, strengthening and weakening of the forces of attraction.

Силы гравитационного притяжения тел играют важную роль как в современной механике и физике, так и в различных областях науки и техники. Эти силы определяются на основе закона притяжения И. Ньютона [1] и развития его представлений в массе тел [2; 3]. Наименее выясненным в законе И. Ньютона остаётся представление о формировании и дальнодействии гравитационного притяжения. Для обхода этих трудностей А. Эйнштейн предложил рассматривать гравитационные эффекты, создаваемые массой, как изменение (искривление) пространства, окружающего массу [4].

Отдельные объяснения связаны с возможным влиянием гравитонов. Как указано в [5], мнение о том, что гравитоны могут передавать гравитационную силу, поддерживается многими физиками, хотя имеются и критические замечания [6]. Они связаны с тем, что оставался не ясным механизм действия гравитонов. В работах [7-9] предложено решение этого вопроса на основании концепции вхождения в массу и последующего реактивного выброса из неё потоков гравитонов и их действия на встречные тела. Рассматриваются тела шаровидной формы. В данной статье представлено развитие этого направления. Учитывается двойственный характер влияния на массу выбросов гравитонов и возможное изменение скорости выброса гравитонов.

Исходные зависимости гравитонной модели и её особое физическое условие (без учёта влияния скорости движения тел, условно в стационарных условиях).
Следуя [7; 8], рассмотрим тело шаровидной формы с центром тяжести в центре шара, обозначим: $r_{1}$ - радиус шара, $m_{1}$ - масса шара, $\Delta m_{1}$ - единичная масса (доля массы шара, отнесенная к единице её поверхности),

$$
\Delta m_{1}=\frac{m_{1}}{4 \pi r_{1}^{2}}
$$

Массу окружают гравитоны, которые входят в неё со скоростью $v_{1}$ и выбрасываются из неё чередующимися всесторонними реактивными потоками со скоростью $V_{1}$ вызывая гравитонное притяжение к ней других масс. В работе [7] рассмотрены иные формирования потоков гравитонов, но все они приводят к одинаковым результатам. Гравитонные потоки оказывают на массу двойное действие. С одной стороны, входя в массу со скоростью $v_{1^{\prime}}$ они её разуплотняют, а с другой стороны, выброс гравитонов из массы приводит к её всестороннему сжатию. Снижение сжатия массы $m_{1}$ вследствие эффекта разуплотнения будем учитывать коэффициентом снижения гравитонного сжатия $Q_{1}$. Силу сжатия $f_{1}$ поверхности единичной массы от выброса гравитонов можно с учётом указанного коэффициента $Q_{1}$ определить из известного из теории реактивных двигателей уравнения, представив его в виде:

$$
f_{1}=\frac{\Delta m_{1}}{\Delta t_{1}} Q_{1} V_{1}=\frac{m_{1} Q_{1} V_{1}}{4 \pi r_{1}^{\bar{z}} \Delta t_{1}}=\frac{\Delta \eta_{1} m_{01} Q_{1} V_{1}}{\Delta t_{1}}=\bar{\eta}_{1} m_{01} Q_{1} V_{1}
$$

где

$$
m_{1}=\Delta \eta_{1} m_{01} 4 \pi r_{1}^{2}
$$

$\Delta \eta_{1}$ - количество гравитонов, выбрасываемых за время $\Delta \mathrm{t}_{1}$ с единицы поверхности шара (общий поток),

$m_{01}$ - включение массы на выброс одного гравитона,

$\bar{\eta}_{1}$ - количество гравитонов, выбрасываемых с поверхности шара за единицу времени (условно единичный поток гравитонов):

$$
\bar{\eta}_{1}=\frac{\Delta \eta_{1}}{\Delta t_{1}}
$$

Используется связь выражения (2) с традиционным проявлением гравитации в виде ускорения $\alpha$, с которым притягиваются тела к центру рассматриваемого шара. Согласно, например, [5], гравитационное ускорение на поверхности шара составляет:

$$
\alpha=\frac{\gamma m_{1}}{r_{1}^{2}}
$$

где $\gamma$ - гравитационная постоянная.

Ускорение (5) вызывает гравитационное сжатие единичной массы силой $f_{1^{\prime}}$ которая, учитывая (1), (5), будет равна:

$$
f_{1}=\Delta m_{1} \cdot \alpha=\frac{\gamma m_{1}^{2}}{4 \pi r_{1}^{4}}
$$


Приравнивая (2) и (6), находим:

$$
f_{1}=\frac{\gamma m_{1}^{2}}{4 \pi r_{1}^{4}}=\frac{\Delta \eta_{1} m_{01} Q_{1} V_{1}}{\Delta t_{1}}=\bar{\eta}_{1} m_{01} Q_{1} V_{1} .
$$

Единичный поток гравитонов будет равен:

$$
\bar{\eta}_{1}=\frac{\gamma m_{1}^{2}}{4 \pi r_{1}^{4} m_{01} Q_{1} V_{1}} .
$$

Сопоставляя (2) и (6) можно заметить, что:

$$
a=\frac{Q_{1} V_{1}}{\Delta t_{1}}=\frac{\gamma m_{1}}{r_{1}^{2}}=\gamma^{*} \Delta m_{1},
$$

где

$$
\gamma^{*}=4 \pi \gamma
$$

Пусть на расстоянии $R$ от тела $m_{1}$ находится тело $m_{2}$ c радиусом $r_{2}\left(R\right.$ - расстояние между центрами тяжести $m_{1}$ и $\left.m_{2}\right)$. При этом в зависимостях (1) - (8) формально индекс «1» заменяется на индекс «2». В результате условие (7) и поток гравитонов $\bar{\eta}_{2}$ в виде (8) для тела $m_{2}$ представляются в виде

$$
\begin{aligned}
& f_{2}=\frac{\gamma m_{2}^{2}}{4 \pi r_{2}^{4}}=\frac{\Delta \eta_{2} m_{02} Q_{2} V_{2}}{\Delta t_{2}}=\bar{\eta}_{2} m_{02} Q_{2} V_{2}, \\
& \bar{\eta}_{2}=\frac{\Delta \eta_{2}}{\Delta t_{2}}=\frac{\gamma m_{2}^{2}}{4 \pi r_{2}^{4} m_{02} Q_{2} V_{2}},
\end{aligned}
$$

где также

$$
m_{2}=\Delta \eta_{2} m_{02} 4 \pi r_{2}^{2}
$$

Рассмотрим определение сил действия потоков гравитонов тела $m_{1}$ на тело $m_{2}$ и наоборот (сил взаимодействия).

При рассмотрении действия потоков гравитонов $\bar{\eta}_{1}$ на тело $m_{2}$ и $\bar{\eta}_{2}$ на тело $m_{1}$ необходимо учитывать три фактора, в первую очередь - фактор их рассеивания. Применительно к рассматриваемой модели на расстоянии $R$ условие рассеивания потоков гравитонов $\bar{\eta}_{1} и \bar{\eta}_{2}$ будет согласно [7] представляться в виде:

$$
\bar{\eta}_{12}=\bar{\eta}_{1} \frac{4 \pi r_{1}^{2}}{4 \pi R^{2}}=\bar{\eta}_{1} \frac{r_{1}^{2}}{R^{2}} ; \quad \bar{\eta}_{21}=\bar{\eta}_{2} \frac{r_{2}^{2}}{R^{2}}
$$

Второй фактор: если потоки тела $m_{1}$ пересекают тело $m_{2} n$ наоборот, то для их полного выброса из масс необходимо, чтобы в зависимостях (3), (13) величины $m_{01}, m_{02}$ изменялись так, что:

$$
m_{1}=\Delta \eta_{2} m^{\prime}{ }_{01} 4 \pi r_{1}^{2}, m_{2}=\Delta \eta_{1} m^{\prime}{ }_{02} 4 \pi r_{2}^{2} \text {, }
$$

где $m_{01}^{\prime}$ - часть массы $m_{1}$ которая включается на выброс одного гравитона из потока $\Delta \eta_{2}$ тела $m_{2}, \mathrm{~m}_{02}^{\prime}$ - аналогично, часть массы тела $m_{2^{\prime}}$ которая включается на выброс одного гравитона $\Delta \eta_{1}$ из потока тела $m_{1}$.

К третьему фактору относятся определение общих потоков гравитонов $\bar{\eta}_{\mathrm{s} 12}$ тела $m_{1}$ и $\bar{\eta}_{\mathrm{s} 21}$ тела $m_{2^{\prime}}$ пересекающих соответственно сферические тела $m_{2}$ и $m_{1}$. Согласно [8]

$$
\begin{gathered}
\bar{\eta}_{s 12}=4 \pi r_{2}^{2} \bar{\eta}_{12}=\bar{\eta}_{1} \frac{r_{1}^{2}}{R^{2}} 4 \pi r_{2}^{2}, \bar{\eta}_{s 21}=4 \pi r_{1}^{2} \bar{\eta}_{21}= \\
\bar{\eta}_{2} \frac{r_{2}^{2}}{R^{2}} 4 \pi r_{1}^{2} .
\end{gathered}
$$

Соответственно общие силы выброса $F_{\text {s12 }}$ и $F_{\text {s21 }}$ гравитонов масс $m_{1} m_{2}$ из масс $m_{1} m_{2}$ по аналогии с (7), (11) представляется в виде:

$$
\left.\begin{array}{c}
F_{12}=\bar{\eta}_{s 12} m_{02}^{\prime} Q_{2} V_{2}=\bar{\eta}_{1} \frac{r_{1}^{2}}{R^{2}} 4 \pi r_{2}^{2} m_{02}^{\prime} Q_{2} V_{2}, \\
-F_{21}=\bar{\eta}_{s 21} m_{01}^{\prime} Q_{1} V_{1}=\bar{\eta}_{2} \frac{r_{2}^{2}}{R^{2}} 4 \pi r_{1}^{2} m_{01}^{\prime} Q_{1} V_{1},
\end{array}\right\}
$$

или учитывая (8), (12)

$$
\left.\begin{array}{c}
F_{12}=\frac{\gamma m_{1}^{2} r_{1}^{2} 4 \pi r_{2}^{2} m_{02}^{\prime} Q_{2} V_{2}}{4 \pi r_{1}^{4} m_{01} Q_{1} V_{1} R^{2}}=\frac{\gamma m_{1}^{2} r_{2}^{2} m_{02}^{\prime} Q_{2} V_{2}}{r_{1}^{2} m_{01} R^{2} Q_{1} V_{1}}, \\
-F_{21}=\frac{\gamma m_{2}^{2} r_{2}^{2} 4 \pi r_{1}^{2} m_{01}^{\prime} Q_{1} V_{1}}{4 \pi r_{2}^{4} m_{02} Q_{2} V_{2} R^{2}}=\frac{\gamma m_{2}^{2} r_{1}^{2} m_{01}^{\prime} Q_{1} V_{1}}{r_{2}^{2} m_{02} R^{2} Q_{2} V_{2}} .
\end{array}\right\}
$$

Из зависимостей (3), (13) и (15) следует

$$
\left.\begin{array}{l}
\frac{m_{2}}{m_{1}}=\frac{\Delta \eta_{1} m_{02}^{\prime} 4 \pi r_{2}^{2}}{\Delta \eta_{1} m_{01} 4 \pi r_{1}^{2}}=\frac{m_{02}^{\prime} r_{2}^{2}}{m_{01} r_{1}^{2}}, \\
\frac{m_{1}}{m_{2}}=\frac{\Delta \eta_{2} m_{01}^{\prime} 4 \pi r_{1}^{2}}{\Delta \eta_{2} m_{02} 4 \pi r_{2}^{2}}=\frac{m_{01}^{\prime} r_{1}^{2}}{m_{02} r_{2}^{2}} .
\end{array}\right\}
$$

С учетом (19) зависимости (18) преобразовываются к виду

$$
F_{12}=\frac{\gamma m_{1} m_{2} Q_{2} V_{2}}{R^{2} Q_{1} V_{1}},-F_{12}=\frac{\gamma m_{1} m_{2} Q_{1} V_{1}}{R^{2} Q_{2} V_{2}}
$$

При

$$
Q_{1} V_{1}=Q_{2} V_{2}
$$

приходим к закону тяготения И. Ньютона

$$
F_{12}=-F_{12}=\frac{\gamma m_{1} m_{2}}{R^{2}}
$$

Таким образом, при условиях (21), (22) закон «действие равно противодействию» и закон притяжения И. Ньютона выполняются автоматически при несколько иной, согласно (9), трактовке гравитационного ускорения.

Уравнение гравитонной модели сучетом влияния скорости движения тел

Следуя [9] рассматриваются тела (массы) $m_{1}$ и $m_{2}$ (сокращенно $\left.m_{\mathrm{i}}\right)$, которые переходят к движению соответственно со скоростями $v_{\mathrm{i}}$ относительно своего начального состояния, соответствующего $v_{\mathrm{i}}=0$ (здесь и ниже $i=1,2$ ). Увеличение скорости движения тел приводит к увеличению выбрасываемых из них потоков гравитонов. При этом силы сжатия $f_{\mathrm{i}}$ тел увеличиваются до некоторых значений $\tilde{f}_{\mathrm{i}}$, приводя к увеличению деформаций тел. В результате радиус тел уменьшается до значений $\tilde{r_{i}}$, приводя к изменению единичных масс $\Delta m_{1}$ до значений 


$$
\Delta \tilde{m}_{i}=\frac{m_{i}}{4 \pi \tilde{r}_{i}^{2}}=\Delta m_{i} \frac{r_{i}^{2}}{\tilde{r}_{i}^{2}}
$$

При этом массы $m_{\mathrm{i}}$ не изменяются, происходит только их уплотнение. Примем закономерность изменения радиуса в виде

$$
\begin{gathered}
\tilde{r}_{i}^{2}=r_{1}^{2} \gamma_{i t} \sqrt{1-\frac{v_{1}^{2}}{n_{i}^{n^{2}} \tilde{V}^{2}}}=r_{i}^{2} \beta_{i}, \quad \beta_{i}=\frac{\tilde{r}_{i}^{2}}{r_{i}^{2}}=\gamma_{i t} \sqrt{1-\frac{v_{1}^{2}}{\widehat{V}^{2} \eta_{j}}}=\gamma_{i t} \tilde{\beta}_{i} \\
i=1,2
\end{gathered}
$$

где $\beta_{\mathrm{i}}, \tilde{\beta}_{\mathrm{i}}, n_{\mathrm{i}}$ - обобщенные параметры податливости тел сжатию, на микроуровне $n_{\mathrm{i}}=1$, на макроуровне $n_{\mathrm{i}}$ изменяется, $\hat{V}$ - максимальная скорость выброса гравитонов, параметр $\gamma_{i t}$ указан в (26).

С учетом (24)

$$
\Delta \tilde{m}_{i}=\frac{\Delta m_{i}}{\beta_{i}}
$$

Полагаем, что при этом могут изменяться единичные доли масс $m_{0 \mathrm{i}}$ и время выброса гравитонов $\Delta t_{\mathrm{i}}$ до значений

$$
\tilde{m}_{0 i}=m_{0 i} \gamma_{0 i}, \Delta \tilde{t}_{i}=\Delta t_{i} \gamma_{i t}
$$

где $\gamma_{0 \mathrm{i}}$ и $\gamma_{\mathrm{it}},(i=1,2$,$) - коэффициенты изменения.$

Гравитонное уравнение (2), (4) для движущихся тел представляется в виде

$$
\tilde{f}_{i}=\frac{\Delta \tilde{m}_{i}}{\Delta \tilde{t}_{i}} \widetilde{Q}_{i} \tilde{V}_{i}=\frac{\Delta \widetilde{\eta}_{i} \tilde{m}_{0 i} \tilde{Q}_{i} \widetilde{V}_{i}}{\Delta \tilde{t}_{i}}=\tilde{\eta}_{i} \widetilde{m}_{0 i} \tilde{V}_{i} \tilde{Q}_{i}
$$

где по аналогии с (4) единичный поток гравитонов

$$
\tilde{\eta}_{i}=\frac{\Delta \widetilde{\eta}_{i}}{\Delta \tilde{t}_{i}}
$$

$\tilde{V}_{\text {i }}$ - изменяемая скорость выброса гравитонов из движущихся тел, $\tilde{Q_{\mathrm{i}}}$ - соответственно изменяемый коэффициент гравитационного сжатия.

По аналогии с (6), (8) определяется сила $\tilde{f}_{\mathrm{i}}$ через гравитационное ускорение движущихся тел

$$
\begin{gathered}
a=\frac{\gamma m_{i}}{\tilde{r}_{i}^{2}}=\frac{\gamma m_{i}}{r_{i}^{2} \beta_{i}^{\prime}} \\
\tilde{f}_{i}=\frac{\gamma m_{i}}{\tilde{r}_{i}^{2}} \cdot \frac{\gamma m_{i}}{4 \pi \tilde{r}_{i}^{2}}=\frac{\gamma m_{i}^{2}}{4 \pi \tilde{r}_{i}^{4}}=\frac{\gamma m_{i}^{2}}{4 \pi r_{i}^{4} \beta_{i}^{2}} .
\end{gathered}
$$

Приравнивая (27) и (30), находим

$$
\tilde{\eta}_{i}=\frac{\gamma m_{i}^{2}}{4 \pi \tilde{r}_{i}^{4} \tilde{m}_{0 i} \widetilde{V}_{i} \tilde{Q}_{i}} .
$$

В данном случае по аналогии с (9)

$$
\frac{\tilde{Q}_{i} \widetilde{V}_{i}}{\Delta \ddot{t}_{i}}=\frac{\gamma m_{i}}{\vec{r}_{i}^{2}}=\frac{\gamma m_{i}}{r_{i}^{2} \beta_{i}}
$$

или, дополнительно учитывая (26),

$$
\frac{\tilde{Q}_{i} \widetilde{V}_{i}}{\Delta t_{i} \gamma_{t i}} \beta_{i}=\frac{\gamma m_{i}}{r_{i}^{2}}
$$

Сопоставляя (33) с (10) можно выразить $\tilde{Q}_{\mathrm{i}}, \tilde{V}_{\mathrm{i}}$ через $Q_{\mathrm{i}}, V_{\mathrm{i}}$ :

$$
\frac{\tilde{Q}_{i} \widetilde{i}_{i} \beta_{i}}{\gamma_{t i}}=Q_{i} V_{i} \text { или } \widetilde{Q}_{i} \widetilde{V}_{i}=\frac{Q_{i} V_{i}}{\widetilde{\beta}_{i}}
$$

Определим силы взаимного притяжения тел при больших скоростях их движения.

Для первого тела $m_{1}(i=1)$ и второго тела $m_{0}(i=2)$ потоки $\tilde{\eta}_{1}, \tilde{\eta}_{2}$ согласно (31) будут равны

$$
\tilde{\eta}_{1}=\frac{\gamma m_{1}^{2}}{4 \pi \tilde{r}_{1}^{4} \tilde{m}_{01} \widetilde{V}_{1} \tilde{Q}_{1}}, \tilde{\eta}_{2}=\frac{\gamma m_{2}^{2}}{4 \pi \tilde{r}_{2}^{4} \tilde{m}_{02} \widetilde{V}_{2} \tilde{Q}_{2}} .
$$

При достижении потоками $\tilde{\eta}_{1}$ тела $m_{2}$ и потоками $\tilde{\eta}_{2}$ тела $m_{1}$ они будут изменяться.

$$
\tilde{\eta}_{12}=\tilde{\eta}_{1} \frac{\tilde{r}_{1}^{2}}{R^{2}}, \tilde{\eta}_{21}=\tilde{\eta}_{2} \frac{\tilde{r}_{2}^{2}}{R^{2}} .
$$

Общие потоки, которые обозначим $\tilde{\eta}_{\mathrm{s} 12}, \tilde{\eta}_{\mathrm{s} 21}$, пересекающие полностью поверхности $m_{2}$ и $m_{1}$ по аналогии с (16), будут равны:

$$
\tilde{\eta}_{s 12}=\tilde{\eta}_{12} \cdot 4 \pi \tilde{r}_{2}^{2}, \quad \tilde{\eta}_{s 21}=\tilde{\eta}_{21} \cdot 4 \pi \tilde{r}_{1}^{2}
$$

Сила $\tilde{F}_{12^{\prime}}$ с которой $m_{1}$ действует на тело $m_{2^{\prime}}$ и сила $\tilde{F}_{21^{\prime}}$ c которой, наоборот, $m_{2}$ действует на $m_{1}$, будут равны

$$
\tilde{F}_{12}=\tilde{\eta}_{s 12} \tilde{m}_{02}^{\prime} \tilde{V}_{2} \tilde{Q}_{2},-\tilde{F}_{21}=\tilde{\eta}_{s 21} \tilde{m}_{01}^{\prime} \tilde{V}_{1} \tilde{Q}_{1},
$$

Учитывая (35) - (38), силы притяжения $\tilde{F}_{12}$ и $\tilde{F}_{21}$, можно представить в виде:

$$
\begin{aligned}
& \tilde{F}_{12}=\frac{\gamma m_{1}^{2} \tilde{r}_{1}^{2} 4 \pi \tilde{r}_{2}^{2} \widetilde{m}_{\Theta 2}^{\prime} \widetilde{V}_{2} \tilde{Q}_{2}}{4 \pi \tilde{r}_{2}^{4} \tilde{m}_{01} \widetilde{V}_{1} \tilde{Q}_{1} R^{2}}=\frac{\gamma m_{1}^{2} \tilde{r}_{2}^{2} \widetilde{m}_{\Theta 2}^{\prime} \tilde{Q}_{2} \widetilde{V}_{2}}{\tilde{r}_{1}^{2} \tilde{m}_{02} \widetilde{V}_{1} \tilde{Q}_{1} R^{2}}, \\
& -\widetilde{F}_{21}=\frac{\gamma m_{2}^{2} \tilde{r}_{2}^{4} 4 \pi \tilde{r}_{1}^{2} \tilde{m}_{j 1}^{\prime} \widetilde{V}_{1} \tilde{Q}_{1}}{4 \pi \tilde{r}_{2}^{4} \widetilde{m}_{02} \widetilde{V}_{2} \tilde{Q}_{2} R^{2}}=\frac{\gamma m_{2}^{2} \widetilde{r}_{1}^{2} \widetilde{m}_{C 1}^{r} \widetilde{V}_{1} \tilde{Q}_{1}}{\tilde{r}_{2}^{2} \widetilde{m}_{02} \tilde{Q}_{2} \widetilde{V}_{2} R^{2}} .
\end{aligned}
$$

Здесь также по аналогии с (3), (13), (15), чтобы потоки тела $m_{1}$ полностью выбрасывались из тела $m_{2}$ и, наоборот, необходимо эти зависимости с учётом (27), (38) представлять в виде

$$
\left.\begin{array}{l}
\Delta \tilde{m}_{1}=\frac{m_{1}}{4 \pi \tilde{r}_{1}^{2}}=\Delta \tilde{\eta}_{1} \tilde{m}_{01}=\Delta \tilde{\eta}_{2} \tilde{m}_{01}^{\prime}, \\
\Delta \tilde{m}_{2}=\frac{m_{2}}{4 \pi \tilde{r}_{2}^{2}}=\Delta \tilde{\eta}_{2} \tilde{m}_{02}=\Delta \tilde{\eta}_{1} \tilde{m}_{02}^{\prime} \cdot
\end{array}\right\}
$$

Из зависимостей (40) следует

$$
\left.\begin{array}{l}
\frac{m_{2}}{m_{1}}=\frac{\Delta \widetilde{\eta}_{1} \widetilde{m}_{02}^{\prime} 4 \pi \tilde{r}_{2}^{2}}{\Delta \widetilde{\eta}_{1} \widetilde{m}_{01} 4 \pi \tilde{r}_{1}^{2}}=\frac{\widetilde{m}_{02}^{\prime} \tilde{r}_{2}^{2}}{\widetilde{m}_{01} \tilde{r}_{1}^{2}} ; \\
\frac{m_{1}}{m_{2}}=\frac{\Delta \widetilde{\eta}_{2} \widetilde{m}_{01}^{\prime} 4 \pi \tilde{r}_{1}^{2}}{\Delta \widetilde{\eta}_{2} \widetilde{m}_{02} 4 \pi \tilde{r}_{2}^{2}}=\frac{\widetilde{m}_{01}^{\prime} \tilde{r}_{1}^{2}}{\widetilde{m}_{02} \tilde{r}_{2}^{2}} \cdot
\end{array}\right\}
$$

С учётом (41) зависимости (39) преобразовываются к виду

$$
\widetilde{F}_{12}=\frac{\gamma m_{1} m_{2} \tilde{Q}_{2} \widetilde{2}_{2}}{R^{2} \widetilde{Q}_{1} \widetilde{V}_{1}},-\widetilde{F}_{21}=\frac{\gamma m_{1} m_{2} \tilde{Q}_{1} \widetilde{V}_{1}}{R^{2} \widetilde{Q}_{2} \widetilde{V}_{2}}
$$

Выражая в (42) значения $\tilde{Q}_{\mathrm{i}} \tilde{V}_{\mathrm{i}}$ через $Q_{\mathrm{i}} V_{\mathrm{i}}$ по зависимостям (34) и полагая согласно (21) $V_{1} Q_{1}=V_{2} Q_{2}$, зависимости (42) преобразовываются к виду 


$$
\begin{aligned}
F_{12} & =\frac{\gamma m_{1} m_{2} Q_{2} V_{2} \widetilde{\beta}_{1}}{R^{2} \widetilde{\beta}_{2} Q_{1} V_{1}}=\frac{\gamma m_{1} m_{2} \widetilde{\beta}_{1}}{R^{2} \widetilde{\beta}_{2}}, \\
-F_{21} & =\frac{\gamma m_{1} m_{2} Q_{1} V_{1} \widetilde{\beta}_{2}}{R^{2} \widetilde{\beta}_{1} Q_{2} V_{2}}=\frac{\gamma m_{1} m_{2} \widetilde{\beta}_{2}}{R^{2} \widetilde{\beta}_{1}} .
\end{aligned}
$$

Зависимости (43) приводят к интересным условиям.

Пусть, например, тело $m_{1}$ находится в стационарных условиях, когда $\beta_{1}=1$, а тело $m_{2}$ движется с большой скоростью $v_{2^{\prime}}$ тогда сила притяжения $F_{12}$ возрастает до величины $1 / \tilde{\beta}_{2}$, что равносильно увеличению массы тела $m_{2}$ до величины $m_{2} /$ $\tilde{\beta}_{2}$. При $\tilde{V}=c$, где $c$ - скорость света, это увеличение массы тела совпадает с полученным увеличением массы тела в опытах Лоренца. Формул Лоренца также используется в специальной теории относительности А. Эйнштейна. В представленной гравитонной модели такой эффект достигается без изменения масс тела. Имеются и другие различия. Так, притяжения телом $m_{2}$ тела $m_{1}$ при увеличении скорости $v_{2}$ равносильно согласно (43), наоборот уменьшению массы тела $m_{2}$ до значений $m_{2} \beta_{2}$. Ели тела $m_{1}$ и $m_{2}$ движутся в пространстве с одинаковой большой скоростью $\left(v_{1}=v_{2}\right)$, тогда $\tilde{\beta}_{1} / \tilde{\beta}_{2}=1$ и закон притяжения И. Ньютона начинает соблюдаться в традиционном виде

$$
F_{12}=-F_{21}=\frac{\gamma m_{1} m_{2}}{R^{2}}
$$

то есть также, как если бы оба тела находились в стационарных условиях.

\section{Лumepamypa}

1. Ньютон, И. Математические начала натуральной философии / И. Ньютон. - М. : Наука, 1989. - С. 690.

2. Джемер, М. Понятие массы в классической и современной физике / М. Джемер. -М. : Прогресс, 1967. - С. 256.

3. Забельский, Ф.С. Масса и её измерение / Ф.С. Забельский. - М. : Атомиздат, 1974.

4. Эйнштейн, А. Сущность теории относительности / А. Эйнштейн. - М. : Иностранная литература, 1959. - С. 160.

5. Грин, Б. Ткань космоса: Пространство, время и текстура реальности / Б. Грин. - М. : ЛИБРОКОМ, 2009.

6. Кемпфер, Ф.А. Путь в современную физику / Ф.А, Кемпфер. - М. : Мир, 1972.

7. Карпенко, Н.И. О реактивной природе сил тяжести / Н.И. Карпенко, С.Н. Карпенко // Academia. Архитектура и строительство. - 2014. - № 1. - С. 87-88.
8. Карпенко, Н.И. 0 физической природе формирования и передачи сил тяжести / Н.И. Карпенко, С.Н. Карпенко // Естественные и технические науки. - 2015. - № 4. - С. 26-31.

9. Карпенко, Н.И. 0 двух подходах к определению сил притяжения гравитонной модели и их изменений при больших скоростях движения тел / Н.И. Карпенко, С.Н. Карпенко. // Akademia. Архитектура и строительство. - 2020. - № 4. - С. 70-75.

\section{References}

1. Nyuton Isaak. Matematicheskie nachala natural'noi filosofii [Philosophia Naturalis Principia Mathematica]. Moscow, Nauka Publ., 1989, 690 p. (In Russ.)

2. Dzhemer Maks. Ponyatie massy $v$ klassicheskoi $i$ sovremennoi fizike [Concept of mass in classical and modern physics]. Moscow, Progress Publ., 1967, 256 p. (In Russ.)

3. F.S. Zabel'skii. Massa i ee izmerenie [Mass and its Measurement]. Moscow, Atomizdat Publ., 1974. (In Russ.)

4. A. Einshtein. Sushchnost' teorii otnositel'nosti [The meaning of relativity]. Moscow, Foreghn Literature Publ., 1959, 160 p. (In Russ.)

5. Brain Grin. Tkan' kosmosa: Prostranstvo, vremya i tekstura real'nosti [The Fabric of The Cosmos: Space, Time, and the Texture of Reality]. Moscow, LIBROKOM Publ., 2009. (In Russ.)

6. F. A. Kempfer. Put' v sovremennuyu fiziku [The Fabric of The Cosmos: Space, Time, and the Texture of Reality]. Moscow, Mir Publ., 1972. (In Russ.)

7. Karpenko N.I., Karpenko S.N. N.I. Karpenko, S.N. Karpenko. 0 reaktivnoi prirode sil tyazhesti [On the Reactive Nature of Gravity]. In: Academia. Arkhitektura i stroitel'stvo [Academia. Architecture and Construction], 2014, no. 1, pp. 87-88. (In Russ., abstr.in Engl.)

8. Karpenko N.I., Karpenko S.N. 0 fizicheskoi prirode formirovaniya i peredachi sil tyazhesti [On physical Nature of Generating and Transmitting Gravity Forces]. In: Estestvennye i tekhnicheskie nauki [Natural and technical sciences], 2015, no. 4, pp. 26-31. (In Russ.)

9. Karpenko N.I., Karpenko S.N. 0 dvukh podkhodakh k opredeleniyu sil prityazheniya gravitonnoi modeli i ikh izmenenii pri bol'shikh skorostyakh dvizheniya tel [0n Two Approached to Determining the Gravitational Forces of the Graviton Model and Its Changeset at High Speeds of Motion of Bodies]. In: Academia. Arkhitektura i stroitel'stvo [Academia. Architecture and Construction], 2020, no. 4, pp. 70-75. (In Russ., abstr. in Engl.) 